(An ISO 3297: 2007 Certified Organization)

Vol. 4, Issue 3, March 2015

\title{
Energy Detection Based Periodic Dynamic Spectrum Sensing Using Cognitive Radio
}

\author{
Shreema Manna (Bera) \\ Assistant Professor, Dept. of ECE, Sikkim Manipal Institute of Technology, Sikkim, India
}

\begin{abstract}
In today's world, with rapidly growing mobile data traffic, our primary objective is to build a smart wireless communication system that is cognizant of its surroundings and adjusts statistical parameters of its input in order to best utilize the spectrum. Cognitive Radio offers a solution to spectrum scarcity by utilizing the spectrum holes. In this paper, I have considered a network operating in a decentralized cooperative manner. Thus, informationbearing signals can hop from one node of the network to a neighbouring node, thereby facilitating communication across the entire spectrum. Here periodic dynamic spectrum-sensing using energy detection method is proposed because of its low computational and implementation complexity. In the proposed method the whole spectrum range is divided among five licensed or primary users. Whenever a slot is empty, a secondary user is assigned that slot. So, at most total five numbers of secondary user can be accommodated. The spectrum is sensed periodically. After some time period when primary user is activated in that slot, the secondary user is automatically transferred to another vacant slot or if vacant slot is not available, it is deactivated. The simulation is done in MATLAB platform. And the simulated result shows that cognitive radio users can sense the whole spectrum periodically and allocate its spectrum dynamically among the secondary users.
\end{abstract}

KEYWORDS:cognitive radio, dynamic spectrum sensing, spectrum hole, primary user, secondary user.

\section{I.INTRODUCTION}

The ever increasing demand of wireless applications restricts the usage of available radio spectrum which is a limited natural and precious resource. A static spectrum assignment has led to underutilisation of spectrum, as a major portion of the spectrum is not effectively utilised [1]. Cognitive radio is a promising technology to effectively utilize the underutilized band of spectrum, which allows the secondary users to access the frequency spectrum, which is originally licensed to primary users when that spectrum is sensed to be idle [3]. Presently available spectrum sensing techniques can be broadly divided into three categories: energy detection, matched filter detection, and cyclostationary detection. Though the matched filter can achieve coherent detection, energy detection is a non-coherent detection method that uses the energy of the received signal to determine the presence of primary signals. Cyclostationary detection exploits the inherent periodicity in the received signal to detect primary signals with a particular modulation type. In order to mitigate the multi-path fading and shadowing effects, cooperative detection methods among multiple CR users are proposed in [4]. All these detection methods are based on the transmitter detection to determine if a signal from primary transmitter is locally present in a certain spectrum through the local observations of CR users. Among all these available methods, energy detection has been widely applied, since it does not require any a priori knowledge of the primary signals and has much lower complexity. The two most significant challenges with the energy detector based sensing is the selection of the threshold for detecting primary users and inability to differentiate interference from primary user signal. In this method energy of the sensed signal in a specific time interval is calculated and compared with a threshold. If the energy is greater than the threshold then a decision is taken as the spectrum is busy, otherwise the spectrum is idle [5-9]. The secondary user will utilize the spectrum if the spectrum is detected idle. Thus the selection of threshold is a crucial parameter in this energy detection based method for sensing the spectrum. The secondary user can make wrong decision depending on the threshold. Two probabilities are defined, first one is the missed detection probability, which is the probability of interference with the primary user and the second one is detection probability, which denotes the correct detection of busy states. If the secondary user gives wrong alarm on primary user's occurrence, that probability is called false alarm probability [6]. This threshold can be fixed or can be dynamically adjusted. In some cases threshold can be adjusted based on the number of signal samples to achieve desired performance in low SNR region [8]. In other case threshold is controlled according to the transmit power of the secondary user such that secondary user's performance can be enhanced with limited interference to the primary user 


\title{
(9) \\ International Journal of Advanced Research in Electrical, Electronics and Instrumentation Engineering
}

ISSN (Print) : $2320-3765$

ISSN (Online): $2278-8875$

\author{
(An ISO 3297: 2007 Certified Organization)
}

\section{Vol. 4, Issue 3, March 2015}

[9]. Recently, by using a data buffering queue at secondary user transmitter, threshold can be dynamically adjusted according to the queue occupancy to ensure statistical quality of service [10]. In order to avoid interference with the primary users, cognitive radio must sense the presence of primary users as quickly as possible and should remove secondary user from the band instantly. Hence, sensing methods should be able to identify the presence of primary users within certain duration [2]. But, CR users cannot perform the transmission and sensing at the same time. Thus, due to this limitation, CR users require a periodic sensing structure where sensing and transmission operations are performed in a periodic manner with separate sensing period and transmission period. In periodic sensing CR users cannot sense the spectrum in transmission time. Again during the observation period, the transmission of CR users is not allowed, which unavoidably decreases the transmission opportunities of CR users, leading to decreasing sensing efficiency [4]. To avoid interference, the observation time must be long enough to achieve sufficient detection accuracy, i.e., longer observation time leads to higher sensing accuracy, and hence to less interference. But as the observation time turn out to be longer, with in a particular period the transmission time of CR users will be decreased. As a result, while a longer transmission time improves the sensing efficiency, it causes higher interference due to the lack of sensing information.

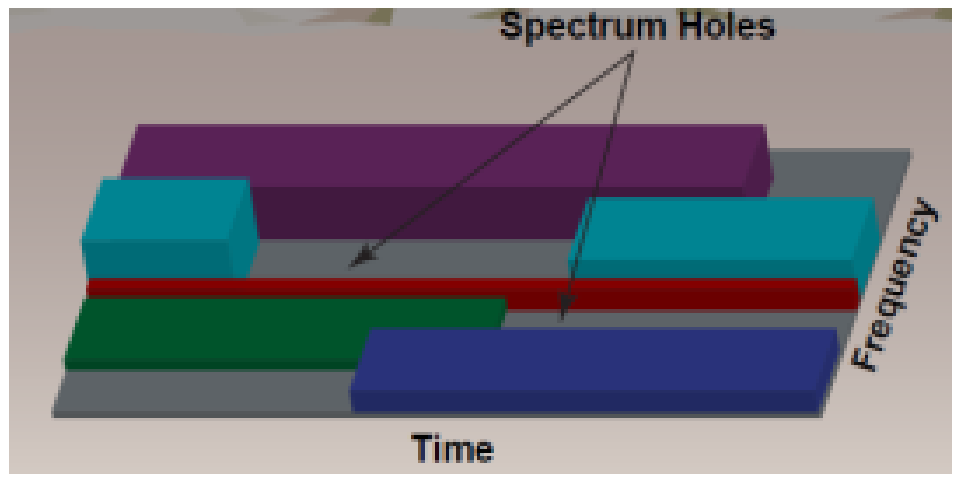

Fig 1: Spectrum holes (the frequency bands that are not occupied by primary user)

\section{RELATED WORKS}

Energy detection, which is a non-coherent detection, is the signal detection mechanism using an energy detector to identify the presence or absence of signal in specific band, which can be implemented in both time domain and frequency domain. This most popular technique for spectrum sensing, the energy detection, where the amount of received energy is the criterion for making a decision regarding the presence of a primary user [6]- [10]. Though this method is easy to implement, it suffers from a relatively poor performance especially for low signal-to- noise ratio (SNR). Author Anirudh M Rao et al. [11] in their paper presented spectrum sensing algorithm under low SNR condition by combining conventional energy detection based technique with statistical principal component analysis (PCA) technique. In [5] author Xiang Ling et al. presented an algorithm to dynamically control the threshold and shows that there is a linear relationship between threshold and SINR (Signal to Interference plus Noise Ratio). Guicai YU et al. [13] investigated the relationship of energy detection performance and detection sensitivity with average noise power fluctuation in short time. The result shows the condition becomes worst in low SNR condition. To improve it they proposed an energy detection algorithm based on dynamic threshold control. BorzooRassouli et al. [2] in their paper considered one secondary user find its optimal sensing parameter after sensing the radio spectrum to maximize its throughput in a periodic sensing framework, while keeping the interference to primary user below a certain level. To this end, they proposed a new interference measure which takes into account the probability of detection, secondary user transmission time and probability of false alarm. In [12], author investigated spectrum sensing algorithms for CRs that support quality-of-service (QoS) traffic. In the proposed scheme multiple bands are sensed in parallel to reduce the sensing delay, while ensuring a fixed minimum rate for CR transmissions with a given outage probability. Interference constraints are also imposed to protect primary user transmissions. Both fixed sample size (FSS) as well as sequential sensing algorithms are developed to minimize the sensing delay. In the sequential sensing case, banks of sequential probability ratio tests (SPRTs) are run in parallel to detect PU presence in all bands concurrently. Notably, the parameters for the detectors can be obtained via convex optimization. Numerical tests demonstrate that sequential sensing yields average sensing delays significantly smaller than those of FSS sensing. 


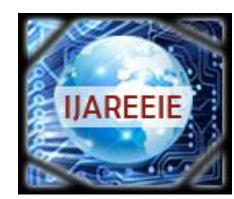

ISSN (Print) : $2320-3765$

ISSN (Online): 2278 - 8875

\section{International Journal of Advanced Research in Electrical, Electronics and Instrumentation Engineering}

(An ISO 3297: 2007 Certified Organization)

Vol. 4, Issue 3, March 2015

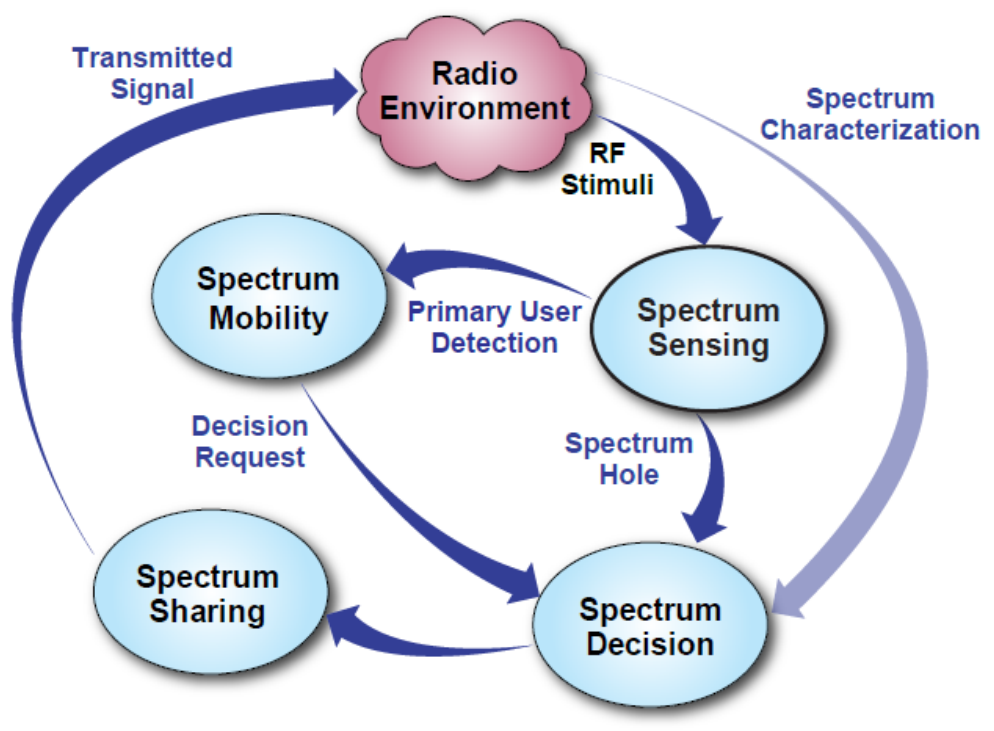

Fig 2: Basic cycle of spectrum sensing and spectrum sharing

\section{III.PROPOSED SYSTEM MODEL AND ASSUMPTIONS}

In this proposed work I have considered a time slotted cognitive radio system consisting of five primary users and five secondary users with an assumption that they use constant power for the transmission of data. The transmitter in the secondary user carries out energy detection based spectrum sensing at the beginning of every time cycle. If the slot is detected idle then the secondary user starts transmitting in that empty slot. The signals from different primary users are amplitude modulated with different carrier signal spread over the spectrum and combined together to cover the whole spectrum. Afterwards power spectral density (using MATLAB command periodogram) of the combined signal and individual primary user signal is determined. Whenever a secondary user wants to transmit, power spectral density of each primary user signal is compared with the threshold and decision is taken accordingly. If the slot is empty, the secondary user signal is amplitude modulated by using corresponding carrier signal and combined with the other active primary user signals to effectively utilize the whole spectrum. After one time period if one or more primary user become active then its corresponding frequency slot is verified to find whether that slot is idle or occupied by some secondary user by sampling the power spectral density of whole spectrum over that corresponding frequency. At the same time secondary user runs the energy detection algorithm to find other vacant slots. If the primary user in previous slot becomes active, that secondary user will automatically be shifted to the next vacant slot, thus making the system dynamic. Here the performance of the system is further investigated by the addition of noise (AWGN) to the combined signal and also by varying signal to noise ratio.

\section{IV.SIMULATION RESULTS AND DISCUSSION}

Simulations are carried out in MATLAB platform. I have set the threshold to a constant value of 8000 and carrier frequencies for five user signals are set as $1 \mathrm{KHz}, 2 \mathrm{KHz}, 3 \mathrm{KHz}, 4 \mathrm{KHz}, 5 \mathrm{KHz}$ and sampling frequency is set to $12 \mathrm{KHz}$. The simulation result in fig 3 depicts active presence of all five primary users. In fig4 shows the absence of primary user 4 and fig 5 shows the assignment of one secondary user in that empty slot. Fig 6, 7 and 8 shows how secondary user is reallocated to the spectrum of second primary user when fourth primary user is activated. 


\title{
International Journal of Advanced Research in Electrical, Electronics and Instrumentation Engineering
}

\author{
(An ISO 3297: 2007 Certified Organization)
}

\section{Vol. 4, Issue 3, March 2015}



Fig3: all five primary users are actively utilizing the spectrum.

The above figure 3 depicts the spectrum when all five primary users are active and thereby occupying the whole spectrum.

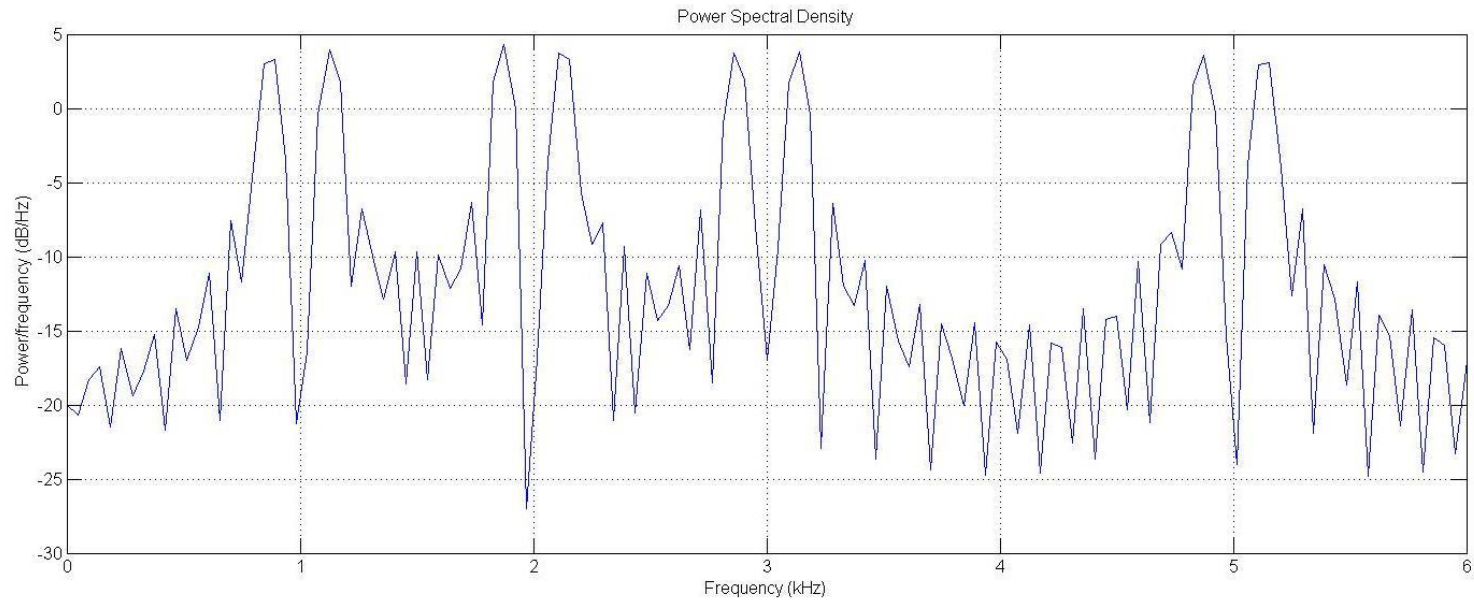

Fig4: fourth user is inactive or idle

Figure 4 represents the spectrum when fourth primary user is not active and its corresponding slot is vacant. This slot can be utilized by other secondary user which wants the access of frequency bands or wants to access the spectrum at this moment. 


\section{International Journal of Advanced Research in Electrical, Electronics and Instrumentation Engineering}

(An ISO 3297: 2007 Certified Organization)

\section{Vol. 4, Issue 3, March 2015}

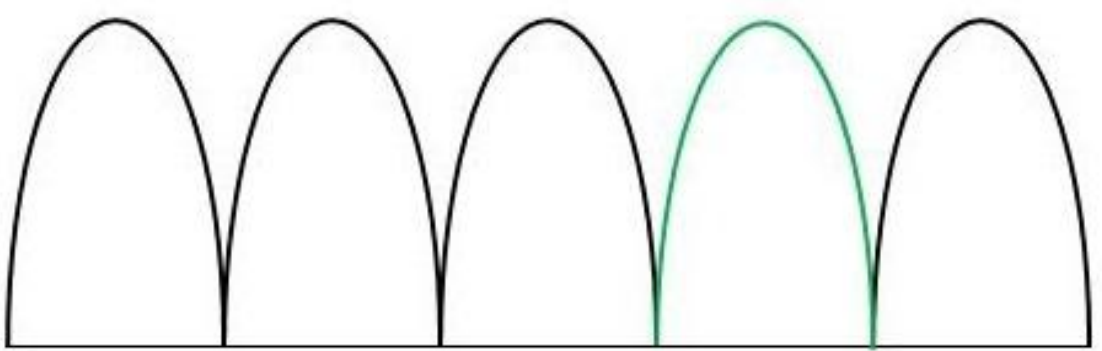

Fig 5: secondary user allocated to the fourth slot

The above figure indicates how one secondary user is assigned the vacant spectrum thereby making effective utilization of the spectrum.

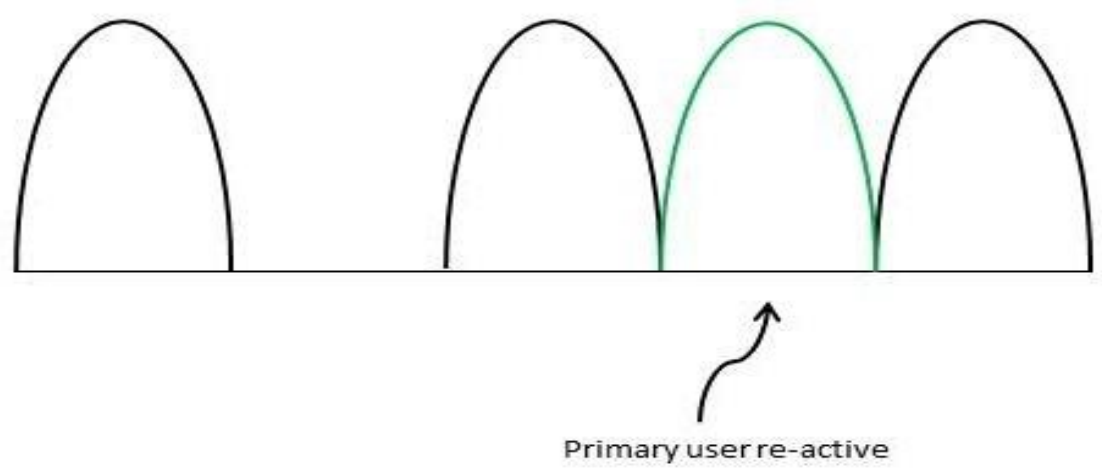

Fig 6: $4^{\text {th }}$ primary user reactivated and $2^{\text {nd }}$ primary user deactivated

Figure 6 depicts the state of the spectrum when second primary user is inactive and fourth primary user becomes reactive again. In this case the secondary user is dynamically assigned the slot of primary user 2 by shifting from the slot of primary user 4 . This is shown in the figure 7 and figure 8 below.

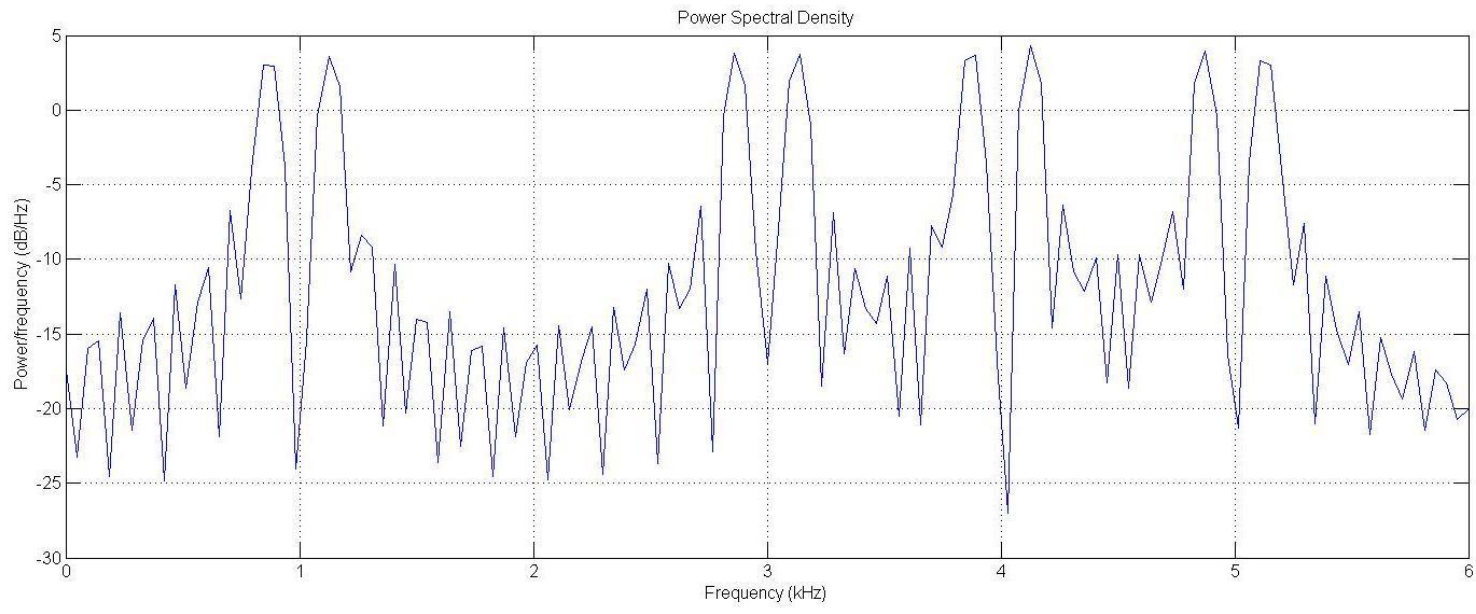

Fig7: second user is absent 


\section{International Journal of Advanced Research in Electrical, Electronics and Instrumentation Engineering}

(An ISO 3297: 2007 Certified Organization)

Vol. 4, Issue 3, March 2015

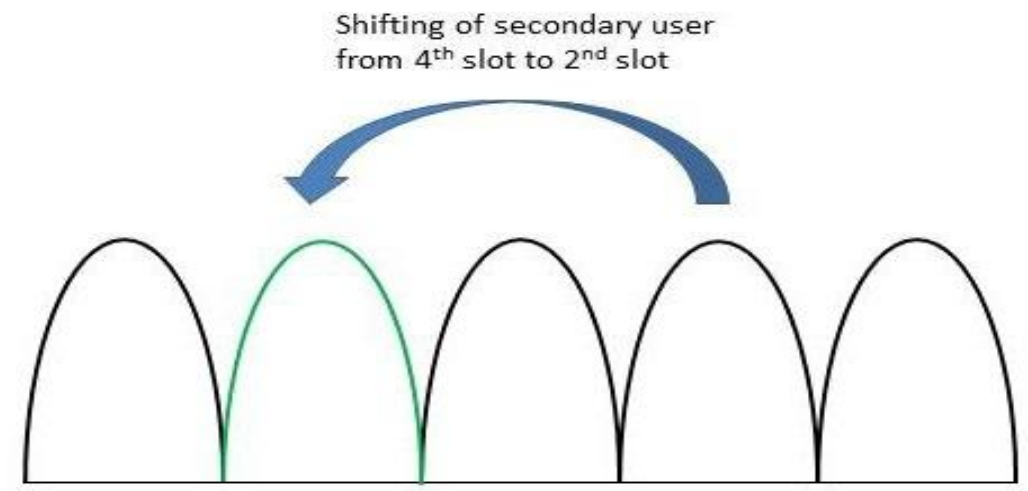

Fig 8: reallocation of secondary user spectrum

\section{V.CONCLUSION}

I have studied dynamic spectrum sensing using energy detection based method in cognitive radios. Here the whole spectrum is periodically sensed and the vacant slot is allocated to secondary user instantly. If primary user in that vacant slot becomes active again, the secondary user is deactivated immediately and reallocated to other vacant slot if it is available. If no slot is vacant secondary user is deactivated.

\section{REFERENCES}

[1] Tevfik Y "ucek and H "useyinArslan, "A survey of spectrum sensing Algorithms for Cognitive Radio Applications", IEEE COMMUNICATIONS SURVEYS \& TUTORIALS, VOL. 11, NO. 1, FIRST QUARTER 2009.

[2] BorzooRassouli and Ali Olfat, "Periodic Spectrum Sensing Parameters Optimization in Cognitive Radio Networks", 19th International Conference on Telecommunications (ICT 2012)

[3] Won-Yeol Lee, and Ian. F. Akyildiz, "Optimal Spectrum Sensing Framework for Cognitive Radio Networks", IEEE TRANSACTIONS ON WIRELESS COMMUNICATIONS, VOL. 7, NO. 10, OCTOBER 2008.

[4] Y.-C. Liang, Y. Zeng, E. C. Y. Peh, and A. T. Hoang, "Sensing-throughput tradeoff for cognitive radio networks," IEEE Trans. Wireless Commun., vol. 7, no. 4, pp. 1326-1337, 2008.

[5] Xiang Ling, Bin Wu, Hong Wen, Pin-Han Ho, ZhiqiangBao and Lili Pan, "Adaptive Threshold Control for Energy Detection Based Spectrum Sensing in Cognitive Radios", IEEE Wireless Communications Letters, VOL. 1, No. 5, October 2012.

[6] P. R. Nair, A. P. Vinod, and A. K. Krishna, "An adaptive threshold based energy detector for spectrum sensing in cognitive radios at low SNR," in Proc. 2010 IEEE International Conference on Communication Systems, pp. 574-578.

[7] H.-H. Choi, K. Jang, and Y. Cheong, "Adaptive sensing threshold control based on transmission power in cognitive radio systems," in Proc. 2008 International Conference on Cognitive Radio Oriented Wireless Networks and Communications, pp. 1-6.

[8] W.-Y. Lee and I. F. Akyildiz, "Optimal spectrum sensing framework for cognitive radio networks," IEEE Trans. Wireless Commun., vol. 7, no. 10, pp. 3845-3857, Oct. 2008.

[9] J. Lee, H. Wang, S. Kim, and D. Hong, "Sensing threshold control for fair coexistence of heterogeneous systems in open spectrum," IEEE Trans.WirelessCommun., vol. 8, no. 12, pp. 5773-5778, 2009.

[10] Q. Du and X. Zhang, "Queue-aware spectrum sensing for interferenceconstrained transmissions in cognitive radio networks," in Proc. 2012 IEEE ICC.

[11] Anirudh M. Rao, B.R. Karthikeyan, DipayanMazumdar, Govind R. Kadambi, "Energy Detection Technique for Spectrum Sensing in Cognitive Radio", SASTECH, vol. 9, Issue 1, April 2010.

[12] Seung-Jun Kim, Guobing Li, and Georgios B. Giannakis, "Multi-Band Cognitive Radio Spectrum Sensing for Quality-of-Service Traffic", IEEE Trans.WirelessCommun, VOL. 10, NO. 10, OCTOBER 2011 